\title{
A Real-Time High Resolution Passive WiFi Doppler- Radar and Its Applications
}

\author{
Bo Tan, Karl Woodbridge \\ Department of Electronic and Electrical Engineering \\ University College London \\ London, UK \\ b.tan@ee.ucl.ac.uk k.woodbridge@ucl.ac.uk
}

\begin{abstract}
The design and implementation of a real-time passive high Doppler resolution radar system is described in this paper. Batch processing and pipelined processing flow are introduced for reducing the processing time to enable real-time display. The proposed method is implemented on a software defined radio (SDR) platform. Two experiments using this system are described: one based on small human body motions and another one on hand gesture detection. The results from these experiments show that the proposed system can be used in a range of application scenarios such as eHealth, human-machine interaction and high accuracy indoor target tracking.
\end{abstract}

\section{Keywords-Passive Radar, Wi-Fi, Micro Doppler, RealTtime}

\section{INTRODUCTION}

Passive radar was first proposed and implemented during World War II for detecting aircraft by using the radio signals emitted by other radar systems [1]. In more recent years, passive systems that used FM radio, analog television and radio communication signals have been developed for detecting and tracking airborne targets. With the deployment of wireless communication infrastructures such as cellular, digital radio and internet networks a number of additional applications have been developed which include passive systems for monitoring highway traffic [2] and Wi-Fi passive radar for indoor person detection [3].

Wi-Fi passive radar has been used for detecting people in office buildings [4] and through-walls [3]. It has also been used for tracking a vehicle in an open field [5]. These studies proved that $802.11 \mathrm{x}$ signals can be used as the illuminator for target detection and tracking in both outdoor and complex indoor scenarios. Recently, further studies in [6], [7] have demonstrated the potential of capturing very small Doppler shifts which can be mapped to small human body movement. The signal processing methods used in [6] and [7] have been out of the scope of traditional radar signal processing. The method for extracting Doppler information in these systems (WiSee and Wi-Vi) is different from the cross correlation methods normally used in radar systems. WiSee developed an equalized FFT method for capturing the Doppler shifts from the reflected Wi-Fi signal, similar to the traditional short-time Fourier transform (STFT). The Doppler resolution of this method depends on the integration time of the FFT. In addition, the complexity of the system using the proposed method increases significantly because it has to implement the demodulation and channel decoding according to $802.11 \mathrm{x}$

\author{
Kevin Chetty \\ Department of Security and Crime Science \\ University College London \\ London, UK \\ k.chetty@ucl.ac.uk
}

protocols for the purpose of the equalizing the OFDM symbols. The Wi-Vi project proposed a 2 TXs and 1 RX system which uses an analog method to extract the Doppler shift from the reflected signal. The analog principle helps the $\mathrm{Wi}-\mathrm{Vi}$ system to work in a real-time mode compared with the STFT or ambiguity function methods. However, the analog processing principle limits the potential of the interference elimination which is very important in an indoor application scenario. The $\mathrm{Wi}-\mathrm{Vi}$ has not been verified with the standard Wi-Fi AP since it uses a specially modified transmitter. Regarding small Doppler shift detection, similar work has been discussed in [8] with an active radar system but little work has been reported in the passive radar field especially passive systems using $\mathrm{Wi}-\mathrm{Fi}$ illuminators. In this paper, a micro-Doppler detection method is proposed for the passive Wi-Fi radar for capturing very small Doppler shifts caused by human body movement.

For a radar system, the detection accuracy is largely determined by two factors: bandwidth; which determines the range resolution; and integration time which determines the Doppler resolution. In a passive system using $\mathrm{Wi}-\mathrm{Fi}$, the signal bandwidth is fixed at $20(802.11 \mathrm{a}, \mathrm{g}, \mathrm{n}) / 22(802.11 \mathrm{~b}) / 40(802.11 \mathrm{n})$ $\mathrm{MHz}$, which equates to 7.5 to 15 meters fix range resolution, while the integration time is variable. This means that the Doppler resolution can be improved by taking longer samples during processing. A similar description can be also found in the other digital Doppler information extraction method, for example more OFDM symbols are required according to the processing principle in [6]. However, the main problem with longer integration times is that a longer processing time is required for extracting the Doppler information from the very large volume of data samples. In some cases, Doppler information may even be lost when the processing time is longer than the next sampling duration. Thus, batch processing [9] is introduced in this paper to reduce the processing time needed for the long samples sequences which are used for high Doppler resolution passive Wi-Fi radar. In addition, a pipeline processing flow is proposed for enabling real-time Doppler output. In this paper we also report the design and build of a real-time software defined radio (SDR) system which has been used to verify the proposed fast cross correlation function (CAF) calculation and related processing flow. Two experiments were designed and carried out for detecting small human body movements and hand gestures. The experimental results prove the effectiveness of the proposed real-time hardware and processing software, demonstrate the potential of real-time high accuracy Doppler tracking and humanmachine interacting based on Wi-Fi signals. 
This paper is organized as follows. Section II describes the proposed real-time processing method and the system configuration. The experiments for small human body motion and hand movements are presented with the results in section III. Finally, the results are summarised in Section 1V and some potential applications discussed.

\section{REAL-TIME SignAL PROCESSING AND SySTEM IMPLEMENTATION}

\section{A. Radar Processing}

In order to obtain the range and Doppler information of target, a 2-D time frequency analysis tool, the cross ambiguity function (CAF) is often used. A discrete form of the CAF is shown in (1):

$$
C A F\left(\tau_{d}, f_{d}\right)=\sum_{n=0}^{N} r[n] s^{*}\left[n+\tau_{d}\right] e^{-j 2 \pi f_{d} \frac{n}{N}}
$$

where $N$ is total number of samples, $n$ is $n$-th sample, $r[n]$ and $s[n]$ are discrete-time reference and surveillance signals in complex form, operator $*$ is complex conjugate of signal, $\tau_{d}$ is time delay which can be converted to range information, $f_{d}$ is Doppler shift.

If the sample rate in the above discrete system is $f_{s} \mathrm{~Hz}$, the integration time of the equation (1) will be $\left(N \cdot f_{S}\right)$ second, the Doppler resolution will be $\left(N \cdot f_{s}\right)^{-1} \mathrm{~Hz}$. Thus, more samples will be required if higher Doppler resolution is needed. For example, a 2 second integration time will be needed for approaching $0.5 \mathrm{~Hz}$ Doppler resolution. However, in some application scenarios such as hand moving or body motion, the target movement velocity and direction will change significantly while the position will be unchanged during the integration time. Then, in one range bin of the CAF surface, more than one Doppler shift will appear. In some particular situations, positive and negative Doppler shifts may appear in the same range bin. In order to reduce the impact from the above phenomenon, a windowing method is often used to obtain the sample sequence to be processed. The windowing method is shown in Figure 1:

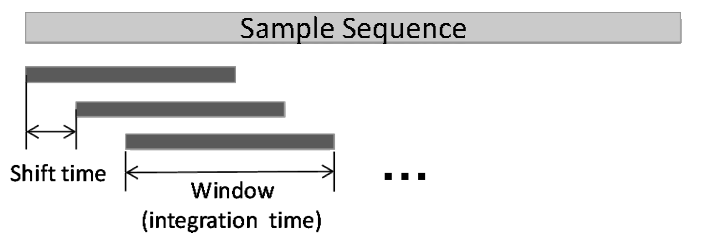

Figure 1. Windowing method

In the windowing method, each Window contains new information comparing with the adjacent previous Window. The processing result with windowing shift will present the clearer Doppler varying trend. The processing delay in windowing method should be limited to less than the shift time shown in Figure. 1, which means an even tighter restriction. Similar limits exist in other digital Doppler shift capturing methods, for example the STFT in [10]. To speed up the Doppler shift capturing processing, batch processing is introduced in this work. The batch processing can be demonstrated by Figure 2 .

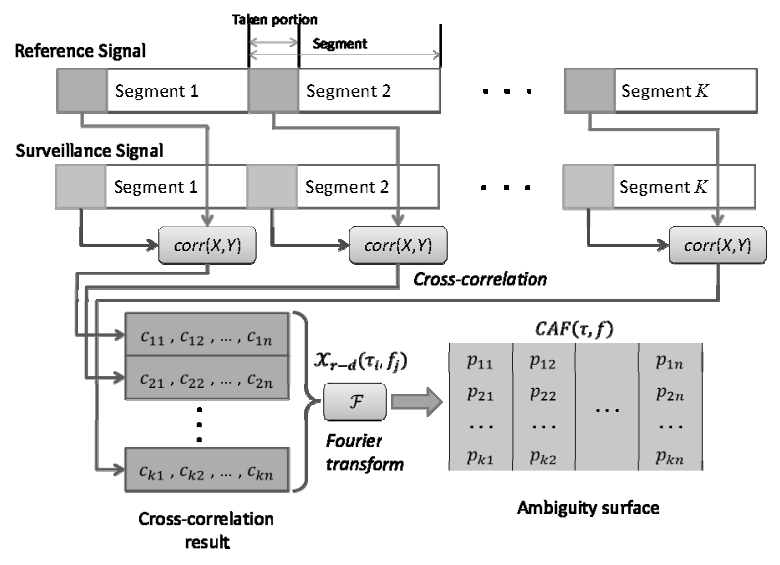

Figure 2. Schematic of batch processing flow

In batch processing, the recorded synchronized reference $r[n]$ and surveillance $s[n]$ signal are first divided into $v$ isometric data segments. For each segment, only a certain portion of the sampled data are taken and used for the followup processing. The experimental results show that taking $10 \%$ portion will be enough to build a clear ambiguity surface. Secondly, the surveillance portion taken is cross-correlated with the corresponding reference portion. Then, $K$ crosscorrelation results $(\operatorname{corr}(X, Y))$ are obtained. The length of the cross-correlated sequence $\operatorname{corr}(X, Y)$ is determined by the maximum bistatic range required in system. Only a few range bins are taken since the range resolution is not the main concern in this work. The cross-correlation results of each portion pair are then combined to form a matrix $X_{r-d}\left(\tau_{j}, f_{i}\right)$ following the method shown in Figure 2. Finally, a Fourier transform is applied on each column of the $\mathcal{X}_{r-d}\left(\tau_{j}, f_{i}\right)$. The ambiguity surface can be demonstrated by the matrix $C A F(\tau, f)$ which is also called cross ambiguity surface.

The number of segments $J$ is determined by the predictable maximum target velocity $v_{\text {max }}$. The relation can be described by the following equation:

$$
J=2 \cdot\left\lceil\frac{v_{\max }}{c} \cdot f_{0} \cdot \tau_{\text {sample }}\right\rceil
$$

where, operator $[\cdot]$ denotes rounding the elements of $X$ to the nearest integers towards infinity, $c$ is the propagation velocity of the wireless signal, and $f_{0}$ is the centre frequency. $\tau_{\text {sample }}$ is the length of the sampled sequence. The factor 2 is used for reserving $v_{\max }$ for both forward and backward directions.

\section{B. Real-time System Design and Implementation}

The application of batch processing significantly reduces the computing time of the CAF. However, as a whole system, other function modules such as signal sampling, interference cancellation are necessary to implement the detection system. Thus, a pipeline processing flow is designed to be implemented on a SDR platform.

1) Pipeline Processing Flow: Pipeline processing is an extension of parallel code execution that enables performance gains with serial multistage algorithms on multiple processing cores. A sequential code sequence is partitioned into subprocedures which are each allocated to a separate processing 
resource. The algorithm can then run simultaneously on multiple sets of recorded data, or data that streams continuously. To maximize throughput each subroutine should be carefully balanced to ensure approximately equal processing delay.

The Virtual Instrument programming environment in LabVIEW employs a structured dataflow design that facilitates pipelined processing. To optimize processing in the wireless passive radar system, the multistage algorithm is divided into three subroutines: (i) sampling the reference and surveillance signal, (ii) performing cross-ambiguity processing on this data using batch processing technique and (iii) applying CLEAN DSI suppression algorithm that are introduced in [11]. Figure 3 illustrates this pipelining strategy.

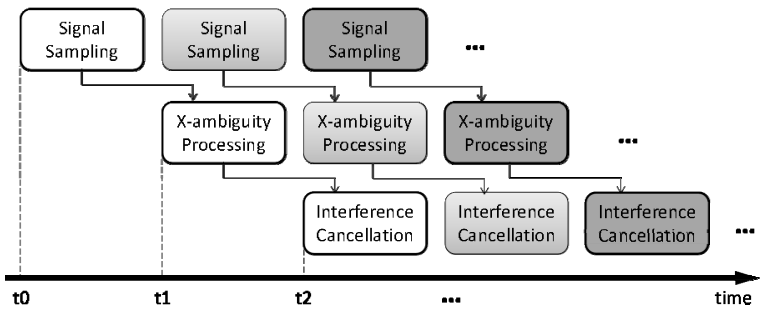

Figure 3. Pipeline processing architecture

The total processing time for the system may be written as

$$
\tau_{P B R}=\max \left\{\tau_{\text {sample }}, \tau_{\text {amb }}, \tau_{\text {cancel }}\right\}
$$

where $\tau_{\text {sample }}$ is the sampling duration which is same as defined in equation (2), $\tau_{a m b}$ and $\tau_{\text {cancel }}$ are the processing delays of subroutines (ii) and (iii). In equation (3), $\tau_{\text {sample }}$ is determined by the system Doppler resolution, cannot be reduce further. Sample delay $\tau_{\text {sample }}$ is equal to Shift time in Figure 1 as the windowing shift method is applied. In order to build a real-time processing flow, $\tau_{a m b}$ and $\tau_{\text {cancel }}$ should not be longer than $\tau_{\text {sample }}$. In practice, interference and noise cancellation algorithms are often simpler, thus, it is easy to control $\tau_{\text {cancel }}$ under $\tau_{\text {sample }}$. The delay of the ambiguity processing involves large volume of long sequence cross correlation and Fourier transforming which cause long processing period, especially the extended sequence, that often exceeds $\tau_{\text {sample }}$. In this work, the batch processing delay $\tau_{a m b}$ is reduced significantly by applying batch processing.

2) Hardware Architecture: Usually, a SDR system consists of antennas, down/up converter and ADC/DAC circuit and a computing platform. In a practical realization of an SDR system, antennas can be chosen in variety of types and frequency bands according to requirement of the application scenarios. Usually the down/up converter function and the $\mathrm{ADC} / \mathrm{DAC}$ function are integrated in one module. A series of commercial circuits can be found for different frequency bands, sampling bandwidth and accuracy. In this paper two synchronized Ettus USRP N210s are chosen for downconverting the RF signal and digitizing. USRP-N210's are each built around a Xilinx ${ }^{\circledR}$ Spartan ${ }^{\circledR}$ 3A-DSP 3400 FPGA and a $100 \mathrm{MS} / \mathrm{s}, 14-$ bit ADC. In receive, the FPGA performs low pass filtering, allowing the recorded data to be transferred via the USRP's gigabit Ethernet port to the host laptop running LabVIEWTM. Each USRP motherboard is also fitted with an RFX2400 daughterboard which can access the spectral band between 2.4-2.9 GHz. The computing unit can be a computing platform with operation system which can implement the signal processing software. Thus, the computing unit can be a PC or embedded chip with a succinct operation system. In this paper, laptop with a 64 bits Windows7 Processional. The laptop is equipped Intel Core i73940XM 3.2 GHz CPU. The operation system provides multiple threads option for implement the pipeline processing in a software platform which is Labview. The architecture of the SDR wireless passive radar is shown in Figure 4.

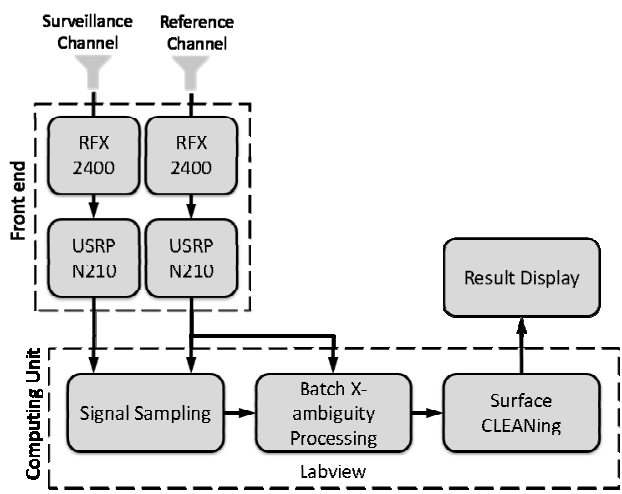

Figure 4. Block diagram of the real-time software-defined wireless passive radar

\section{Doppler Detection Result Display}

Using the above processing flow and computing platform, the Doppler shift can be detected every 0.025 seconds with 0.2 $\mathrm{Hz}$ Doppler resolution. This update rate and Doppler resolution are enough for detecting human body and hand movement. If a higher updating rate and Doppler resolution are required, a more powerful processor would be needed. Each detected Doppler shift is displayed with the corresponding time stamp. Thus, a Doppler record can be generated for each gesture that within the sampling period $\Delta T$. As an example, Figure 5 shows a Doppler record of a torso swaying movement.

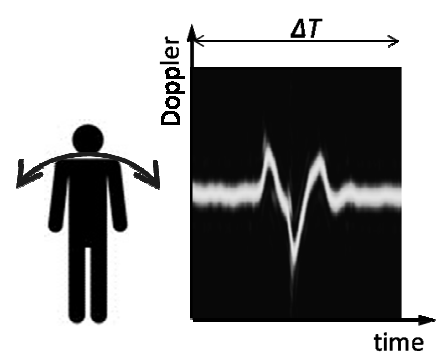

Figure 5. An example of the Doppler record of body swing

\section{EXPERIMENTAL RESULTS}

To verify the effectiveness of the proposed real-time passive detection system, two experiments were designed one for detecting human body gestures in the in-home environment and one for hand movement. In the experiments the small 
Doppler shifts that are caused during the gesture cycle are detected and recorded by the system in real-time. The experimental set-up and results are described in detail in this section.

\section{A. Experiment 1: Human Body Gesture Detection}

The Wi-Fi AP used in this experiment is an Edimax M300 which has $15 \mathrm{dBm}$ power output. One antenna points to the AP as the reference channel and the other antenna is directed to the target as the surveillance channel. Both receivers can be selected from variety of different type of antennas according to the application scenario. For example, for an indoor nonthrough-wall scenario, a low gain, omni-directional antenna is sufficient for detecting the scattered signals. While for a through-wall scenario, high gain antennas are required for optimal performance. The setup of a through-wall human body gesture detection experiment is shown in Figure 6. In this scenario, high gain $(24 \mathrm{dBi})$ dish antennas are used as the reference and surveillance channels.

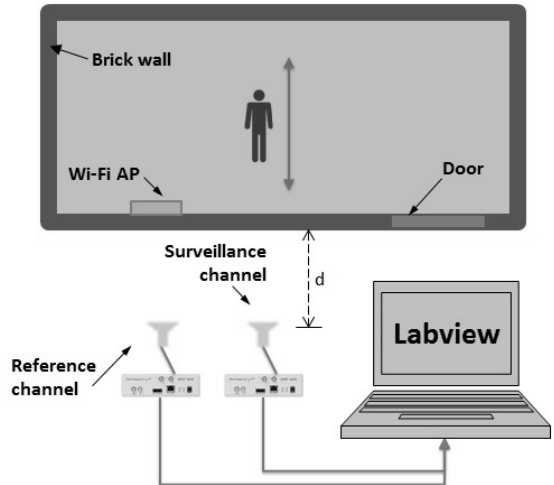

Figure 6 . The experimental setup for through-wall human body gesture detection (thickness of the brick wall is $22 \mathrm{~cm}$ )

In this experiment four different body gestures are tested: stooping-back, squatting-standing, left-right swing and backward-forward swing. The detection results are shown in Figure 7.

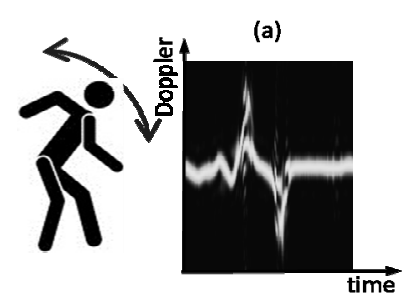

(b)
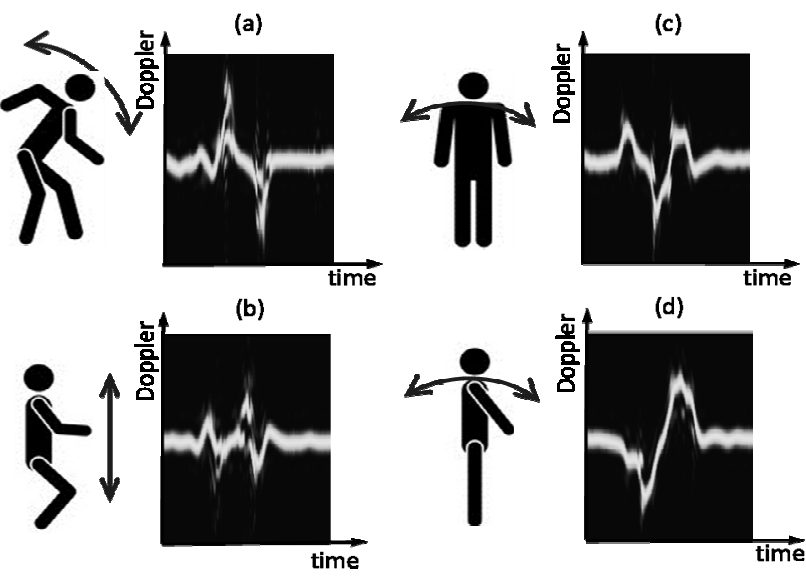

(d)

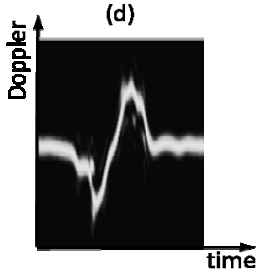

Figure 7. The human body movement results. (a), stooping-back, (b), squating-standing, (c), left-right body swing, (d), forward-backward swing. 4 seconds Doppler record is shown for each guesture cycle. The dynamic Doppler shift range of the body gesture is around $\pm 10 \mathrm{~Hz}$
From the detection results in Figure 7, it can be seen that the real-time Doppler record for each body gesture can be distinguished from the others. In the stooping and squatting experiments (Figure 7a, 7b), single gesture cycle Doppler records are shown. During the stooping-back gesture cycle, only the upper body is approaching or leaving the antenna. Thus, predictably a positive and negative Doppler is generated. The stoop - stand sequence is normally completed quickly as it is a difficult motion to perform slowly. Thus, the Doppler record shows a much sharper characteristic than in the other motions. In addition, the detected signal strengthen is not as strong due to the more limited span of the body movement. Figure 7a clearly shows these Doppler record characteristics. During the squatting-standing gesture cycle, the situation becomes more complex since different parts of the body may have different motions. For example, when squatting down, the knee is moving forward, while the waist is moving backward, meanwhile upper body has slight forward lean. A reverse procedure will occur during the standing period. The Doppler record (Figure $7 b$ ) of this gesture cycle shows a combination of positive and negative excursions. In the left-right swing gesture result, a single cycle gesture Doppler record is shown. In this experiment the subject was facing the wall pointing towards the surveillance antenna on the other side and swinging the torso from left to right. In this geometry the torso movement is angled rather more obliquely towards the bistatic baseline giving slightly smaller bistatic Doppler than the above case and movement occurs in a double cycle. This result in initially positive Doppler followed by significant negative Doppler trough as the torso swings through the complete return cycle. This is then followed by positive Doppler again as the torso swings upright (Figure 7c). The single gesture Doppler record is also shown in the backward-forward swing result. In this case, the upper torso swings directly towards or away from the surveillance antenna at a slight angle to the bistatic baseline. This results in a characteristic sinusoidal positive and negative Doppler which is clearly visible in the Doppler record in Figure $7 \mathrm{~d}$. The value of the detected Doppler shifts is related to the particular geometry of the experiment. The relationship between this Doppler record and experimental geometry can be resolved by the bistatic radar equation and is part of a future study.

\section{B. Experiment 2: Hand Movement Detection}

Many electronic devices such as laptops, tablets and mobile phones can emit Wi-Fi signal and thus could be used as selfilluminator in an integrated passive radar system. To demonstrate this concept, we combined two small receiving antennas and laptop together to set up a miniature passive radar system for capturing the hand movements in front of the screen or around the device. The additional antennas are small size 2.4 $\mathrm{GHz} 2 \mathrm{dBi}$ PCB antennas which are widely used in cell phone, laptop or tablet. As shown in Figure 8, one antenna is located closed to the laptop Wi-Fi which is used as reference channel and the other antenna is fixed at the corner of the keyboard which is used as the surveillance channel. The signals received from the two channels are digitized through the USRPs and sent back to laptop for CAF processing. The laptop used in this experiment is the same as described in the Section II Part B. 


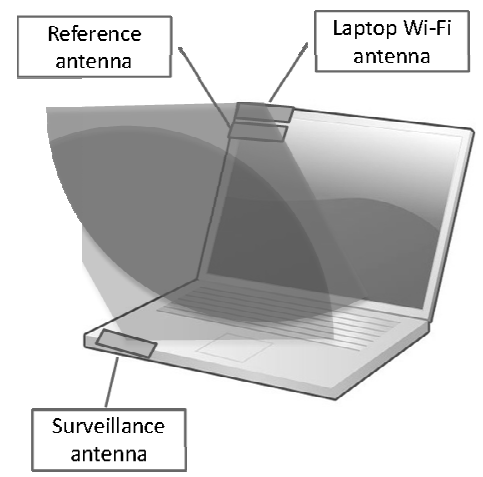

Figure 8. Layout of the hand movement detection system on a laptop

In this paper, four hand movements and the corresponding real-time Doppler records are shown in Figure 9. The examples used in the experiments were forward-backward movements, left-right, counterclockwise and clockwise movements. In this experiment, four hand gestures were carried out continuously. The corresponding real-time Doppler records show clear periodical characteristics. Different hand movements generate different cyclical patterns that are clearly distinguishable. It is noticeable, for example, that the pattern of the counterclockwise hand movement Doppler record is clearly an inverse version of the clockwise hand movement. These preliminary results clearly show that the real-time Doppler detection system has the ability of record the Doppler shifts triggered by different hand movements, which can be used for recognizing hand movement characteristics. In the same way as in the body gesture experiments, the pattern of the Doppler record of different hand gestures depends on experimental geometry. The Doppler pattern will clearly therefore change when the layout geometry is changed. The relation between the Doppler pattern and system geometry will be the subject of the ongoing research into hand gesture recognition using this system.
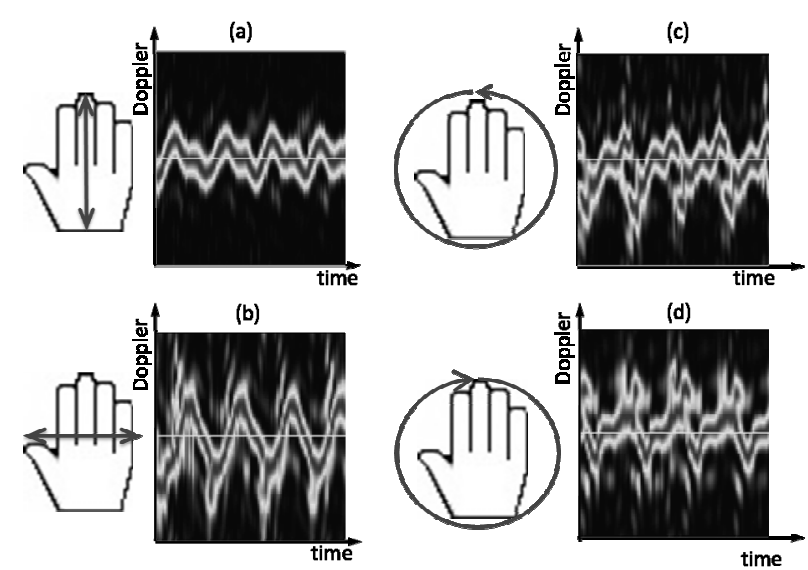

Figure 9. The hand movement detection results. (a), forward-backward moving, (b), left-right moving, (c), counterclockwise moving, (d), clockwise moving. 4 seconds Doppler record is shown for each guesture cycle. The dynamic Doppler shift range of hand moving is around $\pm 5 \mathrm{~Hz}$

\section{CONCLUSIONS}

In this paper, the design, build and experimental testing of a high resolution real time $\mathrm{Wi}-\mathrm{Fi}$ based passive radar has been described. The SDR hardware design enables the development a low cost and adaptable hardware platform. A fast ambiguity processing method is introduced and a pipeline processing flow has been implemented for capturing small Doppler shifts associated with body movements and hand gestures. Real-time body gesture and hand movement experiments have been described and have proved the small Doppler detection method and real-time processing flow work effectively.

Potential applications for this system are widespread and include use as motion sensor in many application scenarios in for example, human-machine interaction (HMI) area, healthcare and location finding fields. In the HMI field, this technology is less complex and easier to implement than other candidate technologies such as infrared in [12] and [13], video based in [14], [15], and wearable sensors as [16]. In healthcare, the system can capture human body gestures and could be used for unobtrusively monitoring the personnel activities of whom in need of guardianship, for example old people or infants. High accuracy indoor tracking could also be implemented using the high resolution Doppler demonstrated and improved localization accuracy in a multiple receiver system.

Future work will concentrate on further experiments in different geometries to build a template of gestures and movements for classification purposes. Various classification methods will be investigated in order to improve the capability of the system. Signal processing developments will include improved DSI and interference suppression and the test and implementation of target tracking algorithms.

\section{REFERENCES}

[1] "Passive Radar." WiKipedia, The Free Encyclopedia. Wikimedia Foundation, Inc. 5 July 2009. Web. 27 Sept. 2013. [Online]. Available: http://en.wikipedia.org/wiki/Passive radar

[2] P. Krysik, P. Samczynski, M. Malanowski, L. Maslikowski, K. S. Kulpa, "Velocity measurement and traffic monitoring using a GSM passive radar demonstrator," IEEE Aerospace and Electronic Systems Magazine, vol.27, no.10, pp.43,51, Oct. 2012

[3] K. Chetty, G.E. Smith, K. Woodbridge, "Through-the-Wall Sensing of Personnel Using Passive Bistatic WiFi Radar at Standoff Distances," IEEE Transactions on Geoscience and Remote Sensing, vol.50, no.4, pp.1218,1226, April 2012

[4] K. Chetty, G.E. Smith, H. Guo, K. Woodbridge, "Target detection in high clutter using passive bistatic WiFi radar," 2009 IEEE Radar Conference, pp.1-5, 4-8 May 2009, Pasadena, CA, USA

[5] P. Falcone, F. Colone, A. Macera, P. Lombardo, "Localization and tracking of moving targets with WiFi-based passive radar," 2012 IEEE Radar Conference, pp.0705-0709, 7-11 May 2012, Atlant, USA

[6] Q. Pu, S. Gupta, S. Gollakota, and S. Patel, "Whole-Home Gesture Recognition Using Wireless Signals", The 19th Annual International Conference on Mobile Computing and Networking (Mobicom'13), September 30-October 4, Miami, FL, USA

[7] F. Adib and D. Katabi, "See Through Walls with Wi-Fi!" ACM Special Interest Group on Data Communications (SIGCOMM'13), Aug 2013, Hong Kong

[8] V.C. Chen, F. Li, S.-S Ho, H. Wechsler, "Analysis of micro-Doppler signatures," IEE Proceedings Radar, Sonar and Navigation, vol.150, no.4, pp.271-6, 1 Aug. 2003

[9] F. Colone, D.W. O'Hagan, P. Lombardo, C.J. Baker, "A Multistage Processing Algorithm for Disturbance Removal and Target Detection in Passive Bistatic Radar," IEEE Transactions on Aerospace and Electronic Systems, vol.45, no.2, pp.698,722, April 2009 
[10] A. Balleri, K. Woodbridge, K. Chetty, "Frequency-agile non-coherent ultrasound radar for collection of micro-Doppler signatures," 2011 IEEE Radar Conference, pp.045-048, 23-27 May 201, Kansas City, MO, USA

[11] K. Chetty, B. Tan, K. Woodbridge, A Real-Time Software Defined Wireless Passive Radar, Radar Conference 2014, Cincinnati, Accepted

[12] "Leap Motion" WiKipedia, The Free Encyclopedia. Wikimedia Foundation, Inc. 4 Oct 2013. Web. 20 Jan. 2014. [Online]. Available: http://en.wikipedia.org/wiki/Leap_Motion

[13] "Kinect" WiKipedia, The Free Encyclopedia. Wikimedia Foundation, Inc. 13 Aug 2013. Web. 20 Dec. 2013. [Online]. Available: http://en.wikipedia.org/w/index.php?title=Kinect\&action=history

[14] PMD Homepage, [Online], Available: http://www.pmdtec.com/

[15] EllipticLabs Homepage, Available: http://www.ellipticlabs.com/

[16] MYO Homepage, Available: https://www.thalmic.com/en/myo/ 